\title{
POWER SERIES THE ROOTS OF WHOSE PARTIAL SUMS LIE IN A SECTOR ${ }^{1}$
}

\author{
LOUIS WEISNER
}

If the roots of the partial sums of a power series $f(z)=\sum a_{n} z^{n}$ lie in a sector with vertex at the origin and aperture $\alpha<2 \pi$, the power series cannot have a positive finite radius of convergence. ${ }^{2}$ But if $f(z)$ is an entire function, the roots of its partial sums may lie in such a sector. The question arises: what restrictions are imposed on $f(z)$ by the requirement that $\alpha$ be sufficiently small, say $\alpha<\pi$ ? According to a theorem of Pólya the order of $f(z)$ must be not greater than 1 if the radius of convergence of the power series is positive. ${ }^{3}$ Without this assumption the investigation which follows shows that if $\alpha<\pi, f(z)$ is an entire function of order 0 . This result was obtained by Pólya for the case in which $\alpha=0 .{ }^{4}$

Lemma. If the complex numbers $z_{1}, \cdots, z_{n}\left(z_{1} \cdots z_{n} \neq 0\right)$ lie in $a$ sector with vertex at the origin and aperture $\alpha<\pi$, then

$$
\frac{n \cos \alpha / 2}{\left|\sum_{k=1}^{n} z_{k}^{-1}\right|} \leqq\left|z_{1} \cdots z_{n}\right|^{1 / n} \leqq \frac{1}{n} \sec \alpha / 2\left|\sum_{k=1}^{n} z_{k}\right| .
$$

When $\alpha=0$ equality occurs if and only if $z_{1}=\cdots=z_{n}$. When $\alpha>0$ equality occurs if and only if $n$ is even and $n / 2$ of the numbers are equal to $r e^{i \phi}(r>0 ; 0 \leqq \phi<2 \pi)$ and the other $n / 2$ numbers are equal to $r e^{i(\phi+\alpha)}$.

Suppose first that the sector is $-\alpha / 2 \leqq$ am $z \leqq \alpha / 2$. Let the $n$ numbers be

$$
z_{k}=\left|z_{k}\right| e^{i \theta_{k}}, \quad-\alpha / 2 \leqq \theta_{k} \leqq \alpha / 2 ; k=1, \cdots, n \text {. }
$$

Since

$$
\sum_{k=1}^{n} z_{k}=\sum_{k=1}^{n}\left|z_{k}\right| \cos \theta_{k}+i \sum_{k=1}^{n}\left|z_{k}\right| \sin \theta_{k}
$$

1 Presented to the Society, April 27, 1940.

2 This follows from Jentzsch's theorem: every point on the circle of convergence of a power series is a limit point of roots of its partial sums. See R. Jentzsch, Untersuchungen zur Theorie der Folgen analytischer Funktionen, Acta Mathematica, vol. 41 (1917), p. 219; E. C. Titchmarsh, Theory of Functions, 1932, p. 238.

${ }^{3} \mathrm{G}$. Pólya, Ueber Annäherung durch Polynome deren sämtliche Wurzeln in einen Winkelraum fallen, Nachrichten der Gesellschaft der Wissenschaften zu Göttingen, 1913, pp. 325-330.

${ }^{4}$ G. Pólya, Ueber Annäherung durch Polynome mit lauter reellen Wurzeln, Rendiconti del Circolo Matematico di Palermo, vol. 36 (1913), pp. 279-295. 


$$
\left|\sum_{k=1}^{n} z_{k}\right| \geqq \sum_{k=1}^{n}\left|z_{k}\right| \cos \theta_{k} \geqq \cos \alpha / 2 \sum_{k=1}^{n}\left|z_{k}\right| \text {. }
$$

Now

$$
\frac{1}{n} \sum_{k=1}^{n}\left|z_{k}\right| \geqq\left|z_{1} \cdots z_{n}\right|^{1 / n} .
$$

Consequently

$$
\frac{1}{n}\left|\sum_{k=1}^{n} z_{k}\right| \geqq \cos \alpha / 2\left|z_{1} \cdots z_{n}\right|^{1 / n} .
$$

Since the numbers $z_{1}^{-1}, \cdots, z_{n}^{-1}$ also lie in the sector $-\alpha / 2 \leqq$ am $z$ $\leqq \alpha / 2$, we have

$$
\frac{1}{n}\left|\sum_{k=1}^{n} z_{k}^{-1}\right| \geqq \cos \alpha / 2\left|z_{1}^{-1} \cdots z_{n}^{-1}\right| 1 / n .
$$

Combining the last two inequalities, (1) results.

When $\alpha=0$, (1) reduces to the well known relation among the harmonic, geometric and arithmetic means of $n$ positive numbers. Here equality occurs if and only if $z_{1}=\ldots=z_{n}$.

If equality occurs in (1) it also occurs in (3) and (4). By (4), $\left|z_{1}\right|$ $=\cdots=\left|z_{n}\right|$. By $(3), \cos \theta_{k}=\cos \alpha / 2$; hence $\theta_{k}= \pm \alpha / 2(k=1, \cdots, n)$. By (2), $\sum_{k=1}^{n} \sin \theta_{k}=0$. Therefore if $\alpha>0, n$ must be even, and $n / 2$ of the numbers equal $r e^{-i \alpha / 2}$, while the other $n / 2$ numbers equal $r e^{i \alpha / 2}$. Conversely, when these conditions are satisfied, equality is attained in (1).

If the numbers are in the sector $\phi \leqq \mathrm{am} z \leqq \mathrm{am}(\phi+\alpha)$, we apply the transformation

$$
z^{\prime}=e^{-i(\alpha / 2+\phi)} z
$$

which rotates this sector into the sector $-\alpha / 2 \leqq \mathrm{am} z \leqq \alpha / 2$ without affecting the value of any member of (1).

Theorem. If, for each $n \geqq n_{0}$, the roots of the partial sum of degree $n$ of the formal power series $f(z)=\sum_{n=0}^{\infty} a_{n} z^{n}$ lie in some sector with vertex at the origin and aperture $\alpha<\pi,{ }^{5}$ then $f(z)$ is an entire function of order 0 .

The case in which $f(z)$ is a polynomial is trivial and is excluded from

\footnotetext{
${ }^{5}$ While $\alpha$ is independent of $n$, we do not require that there shall be one sector which contains the roots of all the partial sums of degree $n \geqq n_{0}$; the lines bounding the sector may be different for different values of $n$.
} 
consideration. We shall suppose $a_{0} \neq 0$; otherwise a power of $z$ could be removed from $f(z)$ without affecting the theorem. Let

$$
f_{n}(z)=\sum_{k=0}^{n} a_{k} z^{k}, \quad n \geqq n_{0} .
$$

By the Gauss-Lucas theorem the roots of $f_{n}^{\prime}(z)$ are also in the sector which contains the roots of $f_{n}(z)$, and the only roots of $f_{n}^{\prime}(z)$ that lie on the boundary of the sector are multiple roots of $f_{n}(z)$; hence $f_{n}^{\prime}(0) \neq 0$. Repeated applications of this argument yield the result that $a_{k} \neq 0(k=0,1, \cdots)$.

According to the lemma, if $z_{1}, \cdots, z_{n}$ denote the zeros of $f_{n}(z)$,

$$
n c\left|\frac{a_{0}}{a_{1}}\right| \leqq\left|\frac{a_{0}}{a_{n}}\right|^{1 / n} \leqq \frac{1}{n c}\left|\frac{a_{n-1}}{a_{n}}\right|, \quad c=\cos \alpha / 2 ; n \geqq n_{0} .
$$

From the first two members of this inequality it follows that $\left|a_{n}\right|^{-1 / n} \rightarrow \infty$ with $n$. Therefore $f(z)$ is an entire function. If $\rho$ is its order,

$$
\frac{1}{\rho}=\liminf _{n \rightarrow \infty} \frac{\log \left|a_{n}\right|^{-1}}{n \log n}
$$

From the last two members of (5) we have

$$
\begin{aligned}
\frac{1}{n} \log \frac{1}{\left|a_{n}\right|}-\frac{1}{n-1} & \log \frac{1}{\left|a_{n-1}\right|} \\
& \geqq \frac{1}{n(n-1)} \log \left|a_{0}\right|+\frac{1}{n-1} \log n c .
\end{aligned}
$$

Let $m=\max \left(n_{0}, 4\right), n>m$. Substituting $n=m, m+1, \cdots, n$ in $(7)$, and adding, we obtain

$$
\begin{aligned}
\frac{1}{n} \log \frac{1}{\left|a_{n}\right|} \geqq \frac{1}{m-1} \log \frac{1}{\left|a_{m-1}\right|}+ & \log \left|a_{0}\right| \sum_{s=m}^{n} \frac{1}{s(s-1)} \\
& +\sum_{s=m}^{n} \frac{\log s}{s-1}+\log c \sum_{s=m}^{n} \frac{1}{s-1} .
\end{aligned}
$$

Now

$$
\begin{aligned}
\sum_{s=m}^{n} \frac{\log s}{s-1}> & \sum_{s=m}^{n} \frac{\log (s-1)}{s-1}>\frac{1}{2} \log ^{2} n-\frac{1}{2} \log ^{2}(m-1), \\
& \sum_{s=m}^{n} \frac{1}{s-1}>\log n-\log (m-1) .
\end{aligned}
$$


Consequently

(8)

$$
\frac{1}{n} \log \frac{1}{\left|a_{n}\right|}>A+\frac{1}{2} \log ^{2} n+\log c \log n,
$$

where $A$ is bounded as $n \rightarrow \infty$. Comparing (6) and (8), we conclude that $\rho=0$.

Hunter College of the City of New York 